\title{
All-oral combination of oral vinorelbine and capecitabine as first-line chemotherapy in HER2-negative metastatic breast cancer: an International Phase II Trial
}

\author{
N Tubiana-Mathieu, , , P Bougnoux ${ }^{2}$, D Becquart ${ }^{3}$, A Chan ${ }^{4}$, P-F Conte ${ }^{5}$, F Majois ${ }^{6}$, M Espie ${ }^{7}$, M Morand ${ }^{8}$, \\ N Vaissiere ${ }^{8}$ and G Villanova ${ }^{8}$
}

${ }^{\prime} \mathrm{CHU}$ Dupuytren, Limoges, France; ${ }^{2} \mathrm{CHU}$ Bretonneau, Tours, France; ${ }^{3}$ AZ Middelheim, Antwerp, Belgium; ${ }^{4}$ Mount Hospital and Royal Perth Hospital, Perth, Australia; ${ }^{5}$ Policlinico di Modena, Modena, Italy; ${ }^{6}$ Hopital Jolimont, Haine St Paul, Belgium; ${ }^{7}$ Hopital St Louis, Paris, France; ${ }^{8}$ Institut de Recherche Pierre Fabre, Boulogne-Billancourt, France

BACKGROUND: This multicentre, international phase II trial evaluated the efficacy and safety profile of a first-line combination of oral vinorelbine plus capecitabine for women with metastatic breast cancer (MBC).

METHODS: Patients with measurable, HER2-negative disease received, as a first line in metastatic setting, 3-weekly cycles of oral vinorelbine $80 \mathrm{mg} \mathrm{m}^{-2}$ (after a first cycle at 60) on day I and day 8, plus capecitabine $1000 \mathrm{mg} \mathrm{m}^{-2}$ (750 if $\geqslant 65$ years of age) twice daily, on days I-14. Treatment was continued until progression or unacceptable toxicity.

RESULTS: A total of 55 patients were enrolled and 54 were treated (median age: 58.5 years). Most (78\%) had visceral involvement and $63 \%$ had received earlier (neo)adjuvant chemotherapy. The objective response rate (RECIST) in 49 evaluable patients was $51 \%$ (95\% confidence interval $(\mathrm{Cl}), 36-66$ ), including complete response in $4 \%$. The clinical benefit rate (response or stable disease for $\geqslant 6$ months) was $63 \%(95 \% \mathrm{Cl}, 48-77)$. The median duration of response was 7.2 months $(95 \% \mathrm{Cl}, 6.4-10.2)$. After a median follow-up of 41 months, median progression-free survival was 8.4 months ( $95 \% \mathrm{Cl}, 5.8-9.7)$ and median overall survival was 29.2 months $(95 \%$ $\mathrm{Cl}, 18.2-40.1$ ). Treatment-related adverse events were manageable, the main grade 3-4 toxicity was neutropaenia (49\%); two patients experienced febrile neutropaenia and three patients had a neutropaenic infection (including one septic death). A particularly low rate of alopaecia was observed.

CONCLUSION: These results show that the all-oral combination of oral vinorelbine and capecitabine is an effective and well-tolerated first-line regimen for $\mathrm{MBC}$.

British Journal of Cancer (2009) I 0 I, 232-237. doi:I0.1038/sj.bjc.6605 I56 www.bjcancer.com

Published online 7 July 2009

(C) 2009 Cancer Research UK

Keywords: oral therapy; first-line chemotherapy; metastatic breast cancer; HER2 negative

Breast cancer is among the most common cancers in Western countries. Advanced breast cancer is the leading cause of death in women aged from 40 to 54 years. About one in eight Western women will develop breast cancer if they live up to an age of 85 years. Despite improving locoregional and adjuvant treatment, many patients still develop recurrent and/or metastatic breast cancer (MBC) within 10 years and will subsequently die of the disease (Harris et al, 2000).

There is no single standard of care for patients with MBC, as treatment plans require an individualised approach based on multiple factors, including tumour biology, growth rate of disease, presence of visceral metastases, history of earlier therapy and response, risk for toxicity and patient preference (O'Shaughnessy, 2005). Preferred first-line single agents in advanced breast cancer are anthracyclines, taxanes, capecitabine, gemcitabine and

*Correspondence: Dr N Tubiana-Mathieu, CHU Dupuytren, 2 avenue Martin Luther King, Limoges, Cedex 87042, France;

E-mail: nicole.tubiana-mathieu@chu-limoges.fr

Received 4 March 2009; revised I June 2009; accepted 2 June 2009; published online 7 July 2009 vinorelbine (NCCN Guidelines, 2009). More recently, bevacizumab has been evaluated in combination with taxanes with interesting clinical results. With anthracyclines and taxanes being used increasingly in early-stage setting, there is an even greater need for other active options in advanced setting to improve outcomes and/or quality of life. The development of oral chemotherapy formulations should allow a higher efficiency, by providing consistent efficacy and reduced patient constraints.

A phase II trial has evaluated oral vinorelbine (Navelbine Oral) single agent as first-line chemotherapy for locally advanced or metastatic breast cancer, showing an overall response rate of $31 \%$ and a median progression-free survival (PFS) of 17.4 weeks (Freyer et al, 2003). Capecitabine (Xeloda) as a single agent has been largely studied in pre-treated metastatic patients, with a median response rate of $28 \%$ and a median time to progression of 4.7 months (Ershler, 2006). Both agents have been included among commonly used compounds for the treatment of $\mathrm{MBC}$ in ESMO Clinical Recommendations (Kataja and Castiglione, 2008).

After establishing both oral vinorelbine and capecitabine as a standard of care in $\mathrm{MBC}$, the development of the combination 
of both agents has a very strong rationale. Moreover, the two agents have different mechanisms of action, different and acceptable safety profiles and synergistic antitumour activity in preclinical models (Sawada et al, 2002). The combination of intravenous vinorelbine (Navelbine) and capecitabine has shown promising efficacy in MBC (Hess et al, 2004; Ghosn et al, 2006; Iodice et al, 2006; Welt et al, 2006; Palumbo et al, 2008). By combining oral vinorelbine and capecitabine, similar outcomes might be achieved without the burden of intravenous infusion.

Three phase I trials have evaluated the combination of oral vinorelbine and capecitabine in MBC (Kellokumpu-Lehtinen et al, 2006; Nole et al, 2006; Anton et al, 2008). In all these studies, the recommended dose of capecitabine for phase II trials was $1000 \mathrm{mg} \mathrm{m}^{-2}$ twice daily, on days $1-14$. As special caution is needed with capecitabine in combination for elderly patients, the doses administered in this trial were reduced to $750 \mathrm{mg} \mathrm{m}^{-2}$ twice daily, on days $1-14$ if age was $\geqslant 65$ years. Regarding oral vinorelbine, different schedules have been evaluated and, among these options, we evaluated in our trial the schedule of $60 \mathrm{mg} \mathrm{m}^{-2}$ on days 1 and 8 for cycle 1 , with a dose escalation to $80 \mathrm{mg} \mathrm{m}^{-2}$ for subsequent cycles in the absence of grade 3 or 4 haematological toxicity.

This international, open-label, phase II trial was designed to evaluate the activity and safety of an all-oral combination of oral vinorelbine and capecitabine as first-line therapy for patients with HER2-negative MBC.

\section{MATERIALS AND METHODS}

\section{Eligibility criteria}

Eligible patients were female, $\geqslant 18$ years, with documented metastatic breast adenocarcinoma untreated by chemotherapy. Other inclusion criteria included HER2-negative disease (IHC 0-1 or IHC $2+$ confirmed as FISH negative), Karnofsky performance status $\geqslant 70 \%$, at least one measurable lesion according to RECIST criteria (Therasse et al, 2000) and a life expectancy $\geqslant 16$ weeks. Adjuvant or neoadjuvant chemotherapy containing an anthracycline and/or a taxane was allowed if $\geqslant 6$ months had elapsed between the last dose of chemotherapy and documentation of relapse. Earlier hormone therapy for advanced disease was allowed. Patients were required to have adequate bone marrow and hepatic and renal functions, indicated by haemoglobin $\geqslant 10 \mathrm{~g}$ per $100 \mathrm{ml}$, absolute neutrophil count $\geqslant 2 \times 10^{9}$ per 1 , platelet count $\geqslant 100 \times 10^{9}$ per 1 , total serum bilirubin $\leqslant 1.5 \times$ upper normal limit (UNL), AST/ALT $\leqslant 2.5 \times \mathrm{UNL},(\leqslant 3.5 \times \mathrm{UNL}$ in case of liver metastases), alkaline phosphatase $\leqslant 2.5$ UNL (or $\leqslant 5$ UNL for bone metastases) and creatinine clearance $>50 \mathrm{ml} \mathrm{min}^{-1}$ (calculated using the Cockroft and Gault formula). Patients were required to give written informed consent before study-specific procedures were performed and to comply with protocol for the duration of the study.

Patients were ineligible if they had only local relapse, earlier chemotherapy in a metastatic setting, previous exposure to a vinca-alkaloid or capecitabine, serious illness or medical conditions such as cardiac disease, unstable diabetes, uncontrolled hypercalcaemia, severe peripheral neuropathy, active infection or previous organ allograft. Patients were also excluded if they were pregnant or lactating, required a concurrent use of the antiviral sorivudine or a chemically related analogue such as brivudine, had clinical central nervous system (CNS) or leptomeningeal metastases, had a malabsorption disease that may affect absorption or oral chemotherapy, had possible hypersensitivity to fluoropyrimidine therapy, had participated in another clinical trial with any investigational drug within 30 days before study inclusion or had a history of another malignancy except cured basal-cell carcinoma of the skin or excised carcinoma in situ of the cervix.

\section{Primary and secondary end points}

The primary end point of the study was overall response. Overall response was defined as the best confirmed response recorded from the date of registration until the end of study period. Secondary objectives included the evaluation of safety, duration of response, PFS and overall survival.

\section{Treatment plan}

Treatment was provided in 3-weekly cycles. Oral vinorelbine was administered at $60 \mathrm{mg} \mathrm{m}^{-2}$ on days 1 and 8 of the first cycle and escalated to $80 \mathrm{mg} \mathrm{m}^{-2}$ at cycle 2 and subsequent cycles in the absence of grade 3 or 4 haematological toxicity.

Capecitabine was administered at a dose of $1000 \mathrm{mg} \mathrm{m}^{-2}$ twice daily, on days $1-14\left(750 \mathrm{mg} \mathrm{m}^{-2}\right.$ twice daily for patients $\geqslant 65$ years).

Prophylactic oral antiemetic medication with a $5-\mathrm{HT}_{3}$ antagonist was recommended before each oral vinorelbine administration.

Treatment was continued until disease progression, unacceptable toxicity or patient's refusal.

\section{Dose modifications}

Dose adjustments and/or treatment delays could be made in the event of dose-limiting haematological or non-haematological toxicities. If study treatment could not be administered after two delays (meaning 2 weeks) of the theoretical day 1 because of any toxicity, it had to be permanently discontinued. Thus, the maximum interval between the start of one cycle and the next was 5 weeks. If one of the agents had to be permanently discontinued, the patient was withdrawn from the study.

Oral vinorelbine was not administered if patients had grade $\geqslant 2$ neutropaenia, and capecitabine was interrupted in case of grade $\geqslant 3$ neutropaenia. After one episode of grade 3 or 4 neutropaenia, the dose of oral vinorelbine was permanently decreased to $60 \mathrm{mg} \mathrm{m}^{-2}$ for subsequent cycles. Patients experiencing grade 3 or 4 neutropaenia, with or without fever, were allowed to receive a granulocyte colony-stimulating factor (G-CSF) in subsequent cycles at the investigator's discretion. If AST/ALT/alkaline phosphatase increased to $>5.0 \mathrm{UNL}$, or if bilirubin increased to $>1.5 \mathrm{UNL}$, both agents were not administered and a reassessment was carried out a week later. If grade $\geqslant 2$ diarrhoea or hand-foot syndrome occurred, administration of capecitabine was interrupted until it resolved to grade 0 or 1 and doses were decreased by $25 \%$ (if grade 3 ) or $50 \%$ (if grade 4 ) for subsequent cycles.

\section{Study assessments}

Every patient who entered the study underwent baseline assessments, including medical history, physical examination, performance status, HER2 testing (either on the primary tumour or on a metastatic site), electrocardiogram and chest X-ray. Tumour measurements by imaging were determined as clinically indicated by computed tomography (CT), abdominal ultrasound, bone scan and/or brain CT scan (if suspicion of CNS involvement). Complete blood cell counts were carried out within 2 days before each oral vinorelbine administration.

Responses were assessed every two cycles until disease progression, or more frequently if early progression was suspected. The best overall response achieved, according to RECIST criteria, was reported for each patient. A complete response (CR) required a complete disappearance of all lesions, and a partial response (PR) required at least a $30 \%$ decrease of the sum of the longest diameters of target lesions. Both $\mathrm{CR}$ and $\mathrm{PR}$ had to be confirmed at 
least 4 weeks later. Stable disease (SD) was defined as neither sufficient shrinkage to qualify for PR nor sufficient increase to qualify for progressive disease (PD). Progressive disease was defined as at least a $20 \%$ increase in the sum of longest diameters of target lesions and/or the appearance of new lesions. Clinical benefit was defined as patients achieving $\mathrm{CR}, \mathrm{PR}$ or $\mathrm{SD}$, maintained for a minimum of 6 months.

Adverse events and medical history were recorded throughout the study. The severity of adverse events was graded according to NCI common toxicity criteria version 2.0.

\section{Statistical analysis}

The one-sample multiple testing procedure for phase II clinical trials as described by Fleming (1982) was used. This procedure uses the standard single-stage test procedure at the last one of two pre-specified tests, while allowing for early termination (should extreme results be seen) and essentially preserving the size and power of the single-stage procedure.

On the basis of a type I error rate of $5 \%$ and a $95 \%$ power to reject the null hypothesis of a $25 \%$ objective response rate (complete or partial), a sample size of 55 patients was needed.

All treated patients were included in the intent-to-treat (ITT) analysis and were analysed for safety. The evaluable population was defined as all patients eligible for the trial who underwent a full evaluation of target and non-target lesions and had received at least two cycles of study treatment (including patients with PD documented before the second cycle).

Response rate and clinical benefit were tabulated together with 95\% confidence interval (CI), following the exact method. The Kaplan-Meier method was applied to overall survival, PFS and duration of response. Subset analysis (according to baseline characteristics) was performed for response rate.

\section{RESULTS}

\section{Patient characteristics}

Between March 2004 and June 2005, 55 patients with MBC were enrolled from 13 sites in six countries. One patient who entered into the study did not receive study treatment because of the presence of a major exclusion criterion (low creatinine clearance). Five of the remaining 54 patients were not evaluable for response, but were included in the ITT analysis: one patient was not eligible for the study (no measurable disease as defined in the protocol), two patients were not assessable as a result of premature study discontinuation (one patient withdrew from the study after the first therapy administration owing to abdominal pain, the second patient died during the second cycle from sepsis) and two patients having received three and six cycles did not undergo a full evaluation of all target and non-target lesions. Therefore, 49 patients with measurable disease were assessable for disease response per protocol.

The patients' characteristics are described in Table 1. Patients typically had visceral metastases and a good performance status. All had HER2-negative disease. Sixty-three percent of patients had received earlier (neo)adjuvant chemotherapy, including an anthracycline in the majority and a taxane in some cases.

\section{Clinical efficacy}

The objective response rate was $51 \%$ among the 49 evaluable patients (95\% CI, 36.3-65.6), including CRs in 4.1\%. Clinical benefit (CR + PR + SD $\geqslant 6$ months) was 63.3\% (95\% CI, $48.3-$ 76.6). In the ITT population, the objective response rate was $46.3 \%$ (95\% CI, 32.6-60.4). Median time to response was 3.1 months (range 1.3-6.7) and median duration of response was 7.2 months (95\% CI, 6.4-10.2) (Table 2).
Table I Patient characteristics

\begin{tabular}{|c|c|c|}
\hline Characteristics & $\mathbf{N}=\mathbf{5 4}$ & (\%) \\
\hline Median age & 58.5 years & \\
\hline Range & $(31.0-84.0)$ & \\
\hline$<65$ & 32 & 59.3 \\
\hline$\geqslant 65$ & 22 & 40.7 \\
\hline \multicolumn{3}{|l|}{ Karnofsky performance status at baseline } \\
\hline $70 / 80$ & 11 & 20.4 \\
\hline $90 / 100$ & 43 & 79.6 \\
\hline \multicolumn{3}{|l|}{ Hormonal status } \\
\hline $\mathrm{ER}+/ \mathrm{PR}+$ & 29 & 53.6 \\
\hline $\mathrm{ER}+/ \mathrm{PR}-$ & 9 & 16.7 \\
\hline ER-/PR+ & 2 & 3.7 \\
\hline $\mathrm{ER}-/ \mathrm{PR}-$ & 9 & 16.7 \\
\hline ER and/or PR unknown & 5 & 9.3 \\
\hline Prior chemotherapy (early stage) & 34 & 63.0 \\
\hline \multicolumn{3}{|l|}{ Type of Chemotherapy } \\
\hline Anthracycline-based without taxane & 23 & 42.6 \\
\hline Anthracycline+taxane & 6 & 11.1 \\
\hline CMF & 5 & 9.3 \\
\hline Prior hormone therapy & 41 & 75.9 \\
\hline For advanced disease & 27 & 50.0 \\
\hline \multicolumn{3}{|l|}{ Number of metastatic sites } \\
\hline I & 7 & 13.0 \\
\hline 2 & 22 & 40.7 \\
\hline$>2$ & 25 & 46.3 \\
\hline Visceral involvement & 42 & 77.8 \\
\hline \multicolumn{3}{|l|}{ Metastatic sites } \\
\hline Liver/lung metastases & $26 / 25$ & $48.1 / 46.3$ \\
\hline Bone metastases & 33 & 61.1 \\
\hline Skin/soft tissue & $3 / 5$ & $5.6 / 9.3$ \\
\hline Median delay between diagnosis and first relapse & 34.3 months & \\
\hline
\end{tabular}

Table 2 Response to treatment

\begin{tabular}{lcc}
\hline $\begin{array}{l}\text { Objective response rate (RECIST) } \\
\text { Evaluable population }\end{array}$ & $\mathbf{n = 4 9}$ & (\%) \\
\hline CR & 2 & 4.1 \\
PR & 23 & 46.9 \\
Objective response $-\mathrm{CR}+\mathrm{PR}-(95 \% \mathrm{Cl})$ & 25 & $51.0(36.3-65.6)$ \\
$\mathrm{SD}$ & 14 & 28.6 \\
$\mathrm{PD}$ & 10 & 20.4 \\
Clinical benefit (CR+PR+SD $\geqslant 6$ months) $(95 \% \mathrm{Cl})$ & 31 & $63.3(48.3-76.6)$ \\
Median time to response (range) & 3.1 months $(1.3-6.7)$ \\
Median duration of response (95\% Cl) & 7.2 months $(6.4-10.2)$
\end{tabular}

$\mathrm{Cl}=$ confidence interval; $\quad \mathrm{CR}=$ complete response; $\mathrm{PR}=$ partial response; $\mathrm{SD}=$ stable disease.

A subanalysis of responses according to patients' characteristics is shown in Table 3. It is noteworthy that the response rate in patients with liver involvement $(52.2 \%)$ was similar to the rate in the overall population.

After a median follow-up of 41 months, 20 of the 54 patients treated in the study were still alive. Median PFS was 8.4 months 
Table 3 Subanalysis of responses according to patients' characteristics

Overall response rate (RECIST)

All evaluable patients $(n=49)$

Liver metastases $(n=23)$

No prior chemotherapy $(n=16)$

Prior anthracycline-based chemotherapy without taxane $(n=23)$

Prior anthracycline+taxane $(n=5)$

Prior CMF $(n=5)$

Prior hormone therapy $(n=36)$

Triple-negative disease $(n=9)$

$\mathrm{CMF}=$ cyclophosphamide, methotrexate, $5-\mathrm{FU} ; \mathrm{MBC}=$ metastatic breast cancer.

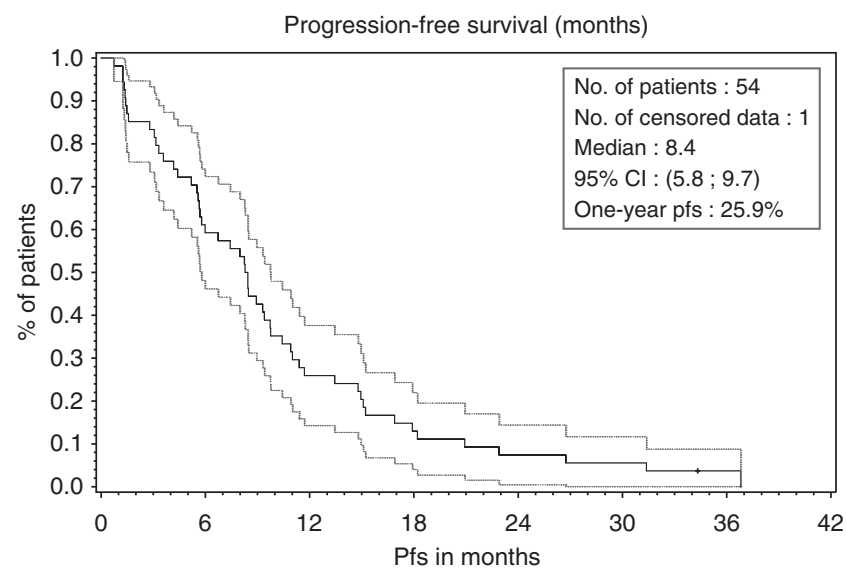

Legend - Survival distribution function E SDF lower $95 \%$ confidence limit SDF upper $95 \%$ confidence limit + + + Censored observations

\begin{tabular}{|l|c|c|c|c|c|c|}
\hline Time (in months) & 6 & 12 & 18 & 24 & 30 & 36 \\
\hline Patients at risk & 32 & 14 & 7 & 4 & 3 & 1 \\
\hline
\end{tabular}

Figure I Progression-free survival (months) intent-to-treat analysis.

(95\% CI, 5.8-9.7 - Figure 1). Median PFS in the ER-positive $(n=39)$ and ER-negative $(n=12)$ population was 8.9 (95\% CI, $6.7-11.0)$ and 4.0 (95\% CI, 1.4-8.2) months, respectively. Median overall survival was 29.2 months (95\% CI, 18.2-40.1 Figure 2).

The majority of patients received further lines of treatment after discontinuation of study therapy: chemotherapy ( $85 \%$ of patients), hormone therapy (54\%) and radiotherapy (33\%).

\section{Drug delivery}

The median number of cycles was seven (range: 1-58). Thirteen patients received study treatment for more than 40 weeks, and eight patients were treated for more than 1 year. The median relative dose intensity of oral vinorelbine and capecitabine was 86.8 and $86.7 \%$, respectively. Dose escalation of oral vinorelbine was achieved in more than $90 \%$ of the patients. In 22 patients $(41 \%)$, the dose of one or both of the agents was reduced. Every oral vinorelbine intake scheduled at day 1 could be administered and only $6.2 \%$ of the doses planned at day 8 had to be omitted.

In eight patients (14.8\%), G-CSF was administered to manage neutropaenia with a curative intent. Two of these patients also received G-CSF with a preventive purpose. In all, G-CSF was administered in 14 cycles $(2.8 \%)$.

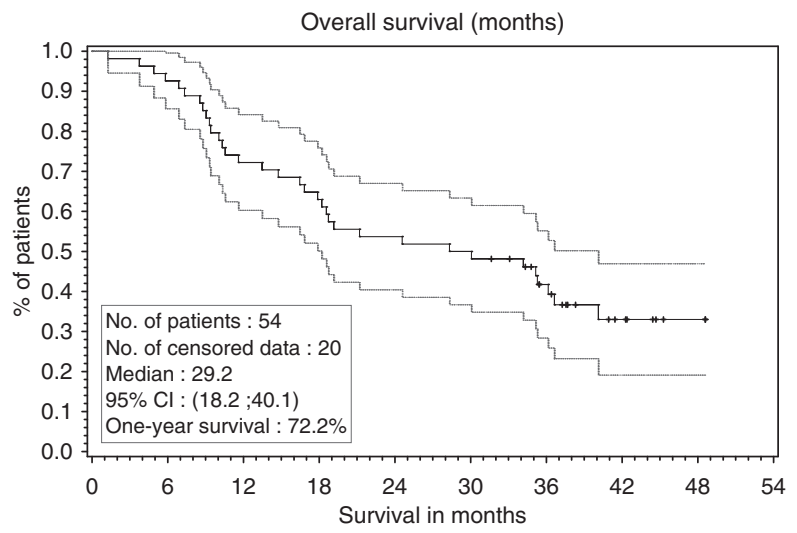

Legend - Survival distribution function E SDF lower $95 \%$ confidence limit

\begin{tabular}{|l|c|c|c|c|c|c|c|c|}
\hline Time (in months) & 6 & 12 & 18 & 24 & 30 & 36 & 42 & 48 \\
\hline Patients at risk & 50 & 39 & 34 & 29 & 27 & 17 & 7 & 2 \\
\hline
\end{tabular}

Figure 2 Overall survival (months) intent-to-treat analysis.

\section{Treatment-related toxicity}

Table 4 shows the incidence of the most common grade $3-4$ side effects related to treatment. The most frequent haematological toxicity was neutropaenia, with grade 3-4 neutropaenia being observed in $49 \%$ of patients. Neutropaenia was usually brief and infrequently associated with infections. Only two patients $(3.8 \%)$ experienced febrile neutropaenia. Three additional patients $(5.6 \%)$ had documented infection associated with grade 3-4 neutropaenia (one fatal septicaemia, one pneumonia and one urinary tract infection). The septic death occurred in a 65-year-old patient with a medical history of non-insulin-dependent diabetes mellitus and a mitral valve replacement who presented, during the second cycle, a positive blood culture in a context of grade 4 neutropaenia and grade 3 diarrhoea.

Non-haematological toxicities were mild. The main grade 3 gastrointestinal adverse event was vomiting, which was seen in $9.3 \%$ of patients and in $1 \%$ of cycles. The incidence of grade $3-4$ diarrhoea was observed in $3.7 \%$ of patients and in $0.6 \%$ of cycles without any grade 4 . Grade 2 alopaecia was observed in $9.3 \%$ and no grade 3-4 neuropathy was observed.

\section{DISCUSSION}

This multicentre study confirms the efficacy of oral vinorelbine with capecitabine previously seen in other studies, with a consistent response rate of more than 50\% (Delcambre et al, 2005; Finek et al, 2009; Nole et al, 2009). The response rate in our trial is comparable with the activity observed with intravenous combinations. Moreover, a similar response rate was achieved in the critical segment of patients having visceral metastases. Disease control was obtained in two-thirds of the patients.

Secondary efficacy end points were also very encouraging: median PFS was 8.4 months and median overall survival was 29.2 months.

There is little evidence from trials reported that major differences exist between many commonly used chemotherapy regimens (Wilcken and Dear, 2008). Among them, the combinations of a taxane with an antimetabolite have been evaluated in two major phase III trials (O'Shaughnessy et al, 2002; Albain et al, 2008). The activity observed with the combination of oral vinorelbine and capecitabine in our trial compares favourably with any of these regimens and provides a strong argument for its development in randomised, comparative trials with any of them. 
Table 4 Treatment-related adverse events

\begin{tabular}{|c|c|c|c|c|}
\hline \multirow[b]{2}{*}{ Adverse events by NCI/CTC v.2.0 } & \multicolumn{2}{|c|}{ Per patient (\%) $N=53^{a}$} & \multicolumn{2}{|c|}{ Per cycle (\%) $N=496$} \\
\hline & Grade 3 & Grade 4 & Grade 3 & Grade 4 \\
\hline Anaemia & 1.9 & 1.9 & 0.2 & 0.2 \\
\hline Leukopaenia & 17.0 & 11.3 & 3.6 & 1.4 \\
\hline Neutropaenia & 26.4 & 22.6 & 6.5 & 3.0 \\
\hline Thrombocytopaenia & 1.9 & 0 & 0.2 & 0 \\
\hline \multirow[t]{3}{*}{ Febrile neutropaenia } & \multicolumn{2}{|c|}{3.8} & \multicolumn{2}{|c|}{0.4} \\
\hline & \multicolumn{2}{|c|}{ Per patient (\%) $N=54$} & \multicolumn{2}{|c|}{ Per cycle (\%) $N=499$} \\
\hline & Grade 3 & Grade 4 & Grade 3 & Grade 4 \\
\hline Nausea & 3.7 & 0 & 0.4 & 0 \\
\hline Vomiting & 9.3 & 0 & 1.0 & 0 \\
\hline Diarrhoea & 3.7 & 0 & 0.6 & 0 \\
\hline Stomatitis & 5.6 & 1.9 & 0.6 & 0.2 \\
\hline Hand-foot syndrome & 3.7 & 0 & 0.8 & 0 \\
\hline Fatigue & 7.4 & 0 & 1.2 & 0 \\
\hline Infection with G3/4 neutropaenia & 3.7 & 1.9 & 0.6 & 0.2 \\
\hline Infection without G3/4 neutropaenia & 3.7 & 0 & 0.4 & 0 \\
\hline Thrombosis/embolism & 1.9 & 1.9 & 0.2 & 0.2 \\
\hline \multirow[t]{2}{*}{ Anorexia } & 1.9 & 0 & 0.4 & 0 \\
\hline & Grade I & Grade 2 & & \\
\hline Alopaecia & 16.7 & 9.3 & & \\
\hline
\end{tabular}

${ }^{\mathrm{a} O n e}$ patient was not evaluable for haematological adverse events

The results of a randomised phase III trial evaluating, as a firstline treatment for HER2-negative MBC patients, the combination of paclitaxel and bevacizumab vs paclitaxel alone have been published recently (Miller et al, 2007). The patient populations entered in this study and in our trial present some similar characteristics (around one-third of patients without any previous chemotherapy and about $10 \%$ of previous anthracycline and taxane in early-stage setting). There is no major difference in the outcome of patients in terms of overall response rate and median overall survival between our trial and the paclitaxel-bevacizumab regimen. Moreover, the selection of patients who may benefit from a bevacizumab-based regimen is not clear yet, this fact being a critical issue because of its high cost.

The toxicities associated with oral vinorelbine and capecitabine were predictable and manageable. As in other phase II studies evaluating this regimen, significant haematological and nonhaematological adverse events were not frequently observed. Nevertheless, as oral chemotherapy is largely taken at home by patients, it is recommended to provide them with adequate information about the measures to be taken in case of gastrointestinal adverse events and to manage them appropriately. The rate of alopaecia in patients receiving this combination was particularly low.

The good tolerability of the combination is apparent from the long duration of therapy in our study. Chemotherapy could be continued until disease progression, with few patients requiring a cessation of treatment because of intolerable adverse effects. The median number of cycles given was seven, and some patients received treatment for more than 1 year. Two meta-analyses comparing longer $v s$ shorter chemotherapy duration in MBC patients have shown that a longer duration was linked with a survival benefit (Coates et al, 2003; Gennari et al, 2008).

Several surveys have shown that, provided efficacy and tolerability are not compromised, most patients prefer oral to intravenous chemotherapy (Liu et al, 1997; Findlay et al, 2008). Oral chemotherapy may reduce anxiety in patients who are afraid of injections. Whether it is administered at home or at the hospital, it reduces treatment-related constraints by requiring fewer and shorter hospital visits and has a smaller impact on daily activities (Findlay et $a l, 2008$ ). Therefore, with a similar efficacy and fewer constraints, this all-oral combination could be considered as being more efficient than intravenous treatments.

In conclusion, the oral vinorelbine/capecitabine combination evaluated in this study is effective and well tolerated. This, together with all the benefits of an oral treatment, the possibility of a longer duration of chemotherapy and of a prolonged infusion-free survival, makes it an attractive regimen that could be proposed as a valid option in first line for HER2-negative, MBC patients.

\section{ACKNOWLEDGEMENTS}

We thank the following investigators of this trial: Vinod Ganju, Frankston Hospital, Frankston, Australia; Miguel Gil Gil, Institut Catala d'Oncologia, L'Hospitalet de Llobregat, Spain; Louis Goedhals, National Hospital, Bloemfontein, South Africa; Antonio Llombart, Instituto Valenciano de Oncologia, Valencia, Spain; Raffaela Palumbo, Fondazione Maugeri, Pavia, Italy and Juan Antonio Virizuela, Hospital Universitario Virgen de la Macarena, Sevilla, Spain. This study has been presented in part at the following conferences: San Antonio Breast Cancer Symposium, San Antonio, Texas, USA, 2006; American Society of Clinical Oncology, Chicago, USA, 2007; European Cancer Conference, Barcelona, Spain, 2007; European Breast Cancer Conference, Berlin, Germany, 2008; European Society of Medical Oncology, Stockholm, Sweden, 2008 and San Antonio Breast Cancer Symposium, San Antonio, Texas, USA, 2008. 


\section{REFERENCES}

Albain K, Nag S, Calderillo-Ruiz G, Jordaan J, Llombart A, Pluzanska A, Rolski J, Melemed A, Reyes-Vidal J, Sekhon J, Simms L, O’Shaughnessy J (2008) Gemcitabine plus paclitaxel $v s$ paclitaxel monotherapy in patients with metastatic breast cancer and prior anthracycline treatment. J Clin Oncol 26: $3950-3957$

Anton A, Lluch A, Casado A, Provencio M, Munoz M, Lao J, Bermejo B, Paules A, Gayo J, Martin M (2008) Phase I-II study of oral vinorelbine and capecitabine in metastatic breast cancer: results of the phase I trial. J Clin Oncol 26: 67s (abstract 1105)

Coates A, Stockler M, Wilcken N (2003) Controversies in Metastatic Breast Cancer: Optimal Duration of Chemotherapy. ASCO Educational Book. pp $119-121$

Delcambre C, Veyret C, Levy C, Switsers O, Allouache D, Raban N, Grellard JM, Leconte A, Delozier T (2005) A phase I/II study of capecitabine combined with oral vinorelbine as first or second line therapy in locally advanced or metastatic breast cancer. Breast Cancer Res Treat 94: S67 (abstract 1061)

Ershler W (2006) Capecitabine monotherapy: safe and effective treatment for metastatic breast cancer. Oncologist 11: 325-335

Findlay M, Von Minckwitz G, Wardley A (2008) Effective oral chemotherapy for breast cancer: pillars of strength. Ann Oncol 19: $212-222$

Finek J, Holubec Jr L, Svoboda T, Sefrhansova L, Pavlikova I, Votavova M, Sediva M, Filip S, Kosevnikova R, Kormunda S (2009) A phase II trial of oral vinorelbine and capecitabine in anthracycline pretreated patients with metastatic breast cancer. Anticancer Res 29: 667-670

Fleming TR (1982) One sample multiple testing procedure for phase II clinical trials. Biometrics 38: $143-151$

Freyer G, Delozier T, Lichinister M, Gedouin D, Bougnoux P, His P, Imadalou K, Trillet-Lenoir V (2003) Phase II study of oral vinorelbine in first-line advanced breast cancer chemotherapy. J Clin Oncol 21: 35-40

Gennari A, Sormani M, Bruzzi N, Wilcken O, Nanni M, Fornier M, Stockler $\mathrm{R}$ (2008) A meta-analysis of chemotherapy duration in metastatic breast cancer. J Clin Oncol 26: 57s (abstract 1067)

Ghosn M, Kattan J, Farhat F, Younes F, Gasmi J (2006) Phase II trial of capecitabine and vinorelbine as first-line chemotherapy for metastatic breast cancer patients. Anticancer Res 26: $2451-2456$

Harris J, Lippmann ME, Morrow M, Osborne CK (2000) Diseases of the Breast, 2nd edn

Hess D, Thurlimann B, Pagani O, Aebi S, Rauch D, Ballabeni P, Rufener B, Castiglione-Gertsch M, Goldhirsch A (2004) Capecitabine and vinorelbine in elderly patients ( $\geqslant 65$ years) with metastatic breast cancer: a phase I trial (SAKK 25/99). Ann Oncol 15: 1760-1765

Iodice G, D'Aiuto G, Comella P, Thomas R, Rubulotta R, Maiorino L, Bianco M, Petrillo A, Frasci G, Comella G (2006) Dose-escalated vinorelbine-capecitabine in elderly metastatic breast cancer patients. A SICOG phase II study. Ann Oncol 17(Supplement 9): ix73 (abstract 155)

Kataja V, Castiglione M (2008) Locally recurrent or metastatic breast cancer: ESMO Clinical Recommendations for diagnosis, treatment and follow-up. Ann Oncol 19(Supplement 2): ii11-ii13
Kellokumpu-Lehtinen P, Sunela K, Lehtinen I, Joensuu H, SjostromMattson J, for the Finnish Breast Cancer Group (2006) A phase I study of an all oral combination of vinorelbine/capecitabine in patients with metastatic breast cancer previously treated with anthracyclines and/or taxanes. Clin Breast Cancer 7: $401-405$

Liu G, Franssen E, Fitch M, Warner E (1997) Patient preferences for oral vs intravenous palliative chemotherapy. J Clin Oncol 15: 110-115

Miller K, Wang M, Gralow J, Dickler M, Cobleigh M, Perez E, Shenkier T, Cella D, Davidson N (2007) Paclitaxel plus bevacizumab vs paclitaxel alone for metastatic breast cancer. NEJM 357: 2666-2676

NCCN Clinical Practice Guidelines in Oncology: Breast Cancer (2009) Version 1.2009

Nole F, Catania C, Sanna G, Imadalou K, Munzone E, Adamoli L, Longerey B, Blanchot G, Goldhirsch A (2006) Dose-finding and pharmacokinetic study of an all-oral combination regimen of oral vinorelbine and capecitabine for patients with metastatic breast cancer. Ann Oncol 17: $322-329$

Nole F, Crivellari D, Mattioli R, Pinotti G, Foa P, Verri E, Fougeray R, Brandely M, Goldhirsch A (2009) Phase II study of an all-oral regimen combining oral vinorelbine with capecitabine as first-line chemotherapy of metastatic breast cancer. Cancer Chemother Pharmacol, DOI 10.1007/ s00280-008-0915-3

O’Shaughnessy J, Miles D, Vukelja S, Moiseyenko V, Ayoub J, Cervantes G, Fumoleau P, Jones S, Lui W, Mauriac L, Twelves C, Van Hazel G, Verma S, Leonard R (2002) Superior survival with capecitabine plus docetaxel combination therapy in anthracycline-pretreated patients with advanced breast cancer: phase III trial results. I Clin Oncol 20: 2812-2823

O'Shaughnessy J (2005) Extending survival with chemotherapy in metastatic breast cancer. Oncologist 10(suppl 3): 20-29

Palumbo R, Bernardo A, Strada M, Teragni C, Poggi G, Frascaroli M, Amatu A, Palumbo I, Bernardo G (2008) Activity and safety of vinorelbine and capecitabine as first-line treatment in patients with metastatic breast cancer - a phase II trial. Eur J Cancer 67 (supplement): 177 (abstract 425)

Sawada N, Fujimoto-Ouchi K, Ishikawa T, Tanaka Y, Ishituka H (2002) Antitumour activity of combination therapy with capecitabine plus vinorelbine, and capecitabine plus gemcitabine in human tumor xenograft models. Proc Am Assoc Cancer Res (abstract 5388)

Therasse P, Arbuck S, Eisenhauer E, Wanders J, Kaplan R, Rubinstein L, Verweij J, Van Glabbeke M, van Oosterom A, Christian M, Gwyther S (2000) New guidelines to evaluate the response to treatment in solid tumors. J Natl Cancer Inst 92: 205-216

Welt A, von Minckwitz G, Oberhoff C, Borquez D, Schleucher R, Loibl S, Harstrick A, Kaufmann M, Seeber S, Vanhoefer U (2006) Phase I/II study of capecitabine and vinorelbine in pretreated patients with metastatic breast cancer. Ann Oncol 16: 64-69

Wilcken N, Dear R (2008) Chemotherapy in metastatic breast cancer: a summary of all randomised trials reported 2000-2007. Eur J Cancer 44: $2218-2225$ 